\title{
Erratum to: The fundamental category of a stratified space
}

\section{Jon Woolf}

Published online: 24 October 2013

(C) Tbilisi Centre for Mathematical Sciences 2013

\section{Erratum to: J. Homotopy Relat. Struct. (2009) 4:359-387}

There is an error in Appendix B (thanks go to Andrei Prasolov for pointing it out). Specifically, Lemma B.3 is false as stated. A further condition is required on a cosheaf to ensure that the basic open sets of its display space are non-empty and connected; we make the following definition.

Definition 0.1 A precosheaf $\mathcal{F}$ is spatial if the basic open sets of its display space $D \mathcal{F}$ are non-empty and connected. Let $\mathbf{S p C o s h} \downarrow \mathbf{X}$ be the full subcategory of spatial precosheaves.

In fact it follows from the remarks in Example B.1 and the proposition below that a spatial precosheaf is a cosheaf.

Proposition 0.2 The following are equivalent for a precosheaf $\mathcal{F}$ :

1. $\mathcal{F}$ is spatial;

2. the counit $C \widehat{D} \mathcal{F} \rightarrow \mathcal{F}$ is an isomorphism;

3. $\mathcal{F} \cong C Y$ for some locally-connected $p: Y \rightarrow X$.

Proof Suppose that $\mathcal{F}$ is a spatial precosheaf. Then the display space $D \mathcal{F}$ is locallyconnected. Hence $D \mathcal{F}=\widehat{D} \mathcal{F}$. Moreover,

$$
C D \mathcal{F}(U)=\pi_{0}\left(p_{D \mathcal{F}}^{-1} U\right)=\pi_{0}\left(\sum_{\alpha \in \mathcal{F}(U)} U(\alpha)\right) \cong \mathcal{F}(U) .
$$

\footnotetext{
J. Woolf $(\bowtie)$

Mathematical Sciences, University of Liverpool, Liverpool L69 7ZX, UK

e-mail: Jonathan.Woolf@liverpool.ac.uk
} 
So the counit $C \widehat{D} \mathcal{F} \rightarrow \mathcal{F}$ is an isomorphism. That the second statement implies the third is clear, since $\widehat{D} \mathcal{F}$ is locally-connected.

To see that the third statement implies the first, suppose, without loss of generality, that $\mathcal{F}=C Y$ for some locally-connected $p: Y \rightarrow X$. Let $U \subset X$ be open and $\alpha \in \mathcal{F}(U)$. The corresponding basic open subset of the display space $D \mathcal{F}$ is $U(\alpha)=$ $\left\{\beta \in \mathcal{F}_{x} \mid x \in U, \beta_{U}=\alpha\right\}$. Consider the unit $\eta_{Y}: Y \rightarrow D C Y=D \mathcal{F}$. It is continuous since $Y$ is locally-connected. Moreover, $\eta_{Y}^{-1} U(\alpha)=\alpha \in \pi_{0}\left(p^{-1} U\right)$ so that it, hence also $U(\alpha)$, is non-empty. Now suppose $U(\alpha)=V^{\prime}+V^{\prime \prime}$ is a disjoint union of open sets. Then $\alpha=\eta_{Y}^{-1} U(\alpha)=\eta_{Y}^{-1}\left(V^{\prime}\right)+\eta_{Y}^{-1}\left(V^{\prime \prime}\right)$ is also a disjoint union of open sets. Since $\alpha$ is a connected component this is only possible if either $\eta_{Y}^{-1}\left(V^{\prime}\right)$ or $\eta_{Y}^{-1}\left(V^{\prime \prime}\right)$ is empty. It follows that either $V^{\prime}$ or $V^{\prime \prime}$ is empty, and therefore that $U(\alpha)$ is connected. Hence $\mathcal{F}$ is spatial.

The next lemma ensures that the cosheaf arising from a representation of the fundamental category is spatial (so that the extra assumption required is always satisfied for the applications in the paper).

Lemma 0.3 Let $G: \Pi_{1}^{o p}(X) \rightarrow$ Set be a functor, and let $\mathcal{G}$ be the precosheaf with cosections $\mathcal{G}(U)=\sum_{x \in U} G(x) / \sim$ where $\sim$ is the equivalence relation generated by $a \sim a^{\prime}$ if there is an op-path $\gamma$ in $U$ from $x$ to $x^{\prime}$ with $a^{\prime}=G(\gamma)(a)$. Then $\mathcal{G}$ is spatial, in particular it is actually a cosheaf.

Proof Using Lemma 4.1 we find that the costalk $\mathcal{G}_{x}=G(x)$. Therefore the display space $D \mathcal{G}$ is $\sum_{x \in X} G(x)$ with the topology generated by the basic open sets $U(\alpha)$ for $\alpha \in \mathcal{G}(U)$ where $U \subset X$ is open. Note that the cosection $\alpha \in \mathcal{G}(U)$ is represented (non-uniquely) by some $a \in G(y)$ where $y \in U$. In terms of this representative, $U(\alpha)=\{b \in G(x) \mid x \in U, b \sim a\}$. It follows that the basic open sets are non-empty and connected. Hence $\mathcal{G}$ is a spatial precosheaf.

This error necessitates the following corrections:

\$1.1 Delete the last two sentences.

p14, line 19 Insert 'spatial' before 'cosheaves' (so that a constructible cosheaf is defined to be spatial).

p14, line 31 Insert 'spatial' before 'cosheafification'.

p14, line 37 Insert 'spatial' before 'cosheaf'.

Lemma B.3 and Remark B.4 These are replaced by the Definition 0.1, Proposition 0.2 , and Lemma 0.3 above.

Corollary B.5 and Proposition B.6 Insert 'spatial' before 'cosheaf' wherever it appears in statements and proofs.

p29, line 15 Insert 'spatial' before 'cosheaves'.

p29, diagram and Remark B.7 Replace $\operatorname{Cosh} \downarrow \mathbf{X}$ by SpCosh $\downarrow \mathbf{X}$ and insert 'spatial' before each occurrence of 'precosheaf', 'cosheaf', and 'cosheafification'. 


\section{Reference}

1. Woolf, J.: The fundamental category of a stratified space. J. Homotopy Relat. Struct. 4, 359-387 (2009) 